\title{
L'Imam, le conférencier et le jurisconsulte : retour sur trois figures contemporaines du champ religieux islamique en France
}

Franck Frégosi

\section{(2) OpenEdition \\ Journals}

Édition électronique

URL : http://journals.openedition.org/assr/1040

DOI : 10.4000/assr. 1040

ISSN : $1777-5825$

Éditeur

Éditions de l'EHESS

Édition imprimée

Date de publication : 1 janvier 2004

Pagination : 131-146

ISBN : 2-222-96741-4

ISSN : 0335-5985

Référence électronique

Franck Frégosi, «L'Imam, le conférencier et le jurisconsulte : retour sur trois figures contemporaines du champ religieux islamique en France », Archives de sciences sociales des religions [En ligne], 125 | janvier - mars 2004, mis en ligne le 22 février 2007, consulté le 19 avril 2019. URL : http:// journals.openedition.org/assr/1040; DOI : 10.4000/assr.1040 


\section{L'IMAM, LE CONFÉRENCIER ET LE JURISCONSULTE : RETOUR SUR TROIS FIGURES CONTEMPORAINES DU CHAMP RELIGIEUX ISLAMIQUE EN FRANCE}

L'étendue du champ du leadership religieux musulman en France peut être appréhendé à l'aune de trois dynamiques majeures qui contribuent empiriquement à en définir les limites selon des lignes fluctuantes. Celles-ci sont en partie indexées sur le devenir de l'institutionnalisation de l'islam et sur la délimitation d'une islamité compatible avec la revendication d'une citoyenneté active.

C'est ainsi que l'on assiste conjointement :

- à des tentatives plus ou moins artificielles de structuration du champ de l'autorité religieuse musulmane sous la forme de processus de rationalisation progressive de l'encadrement religieux musulman national avec pour corollaire une valorisation de la figure de l'imam d'une part;

- à l'émergence progressive de nouveaux types de clercs dont l'essentiel de l'autorité découle moins d'un savoir livresque classique que d'une expérience individuelle militante au nom de la foi et de la revendication d'un engagement citoyen au nom de l'islam;

- enfin se multiplient diverses tentatives individuelles ou organisationnelles visant cette fois à apporter des réponses légales (par rapport à la législation islamique) aux interrogations des fidèles en matière de pratique de l'islam dans des sociétés non musulmanes et sécularisées.

Ces dynamiques nous conduisent à nous interroger sur les divers canaux et les lieux d'expression de l'autorité religieuse au sein de collectivités musulmanes minoritaires et ne pouvant s'adosser à la puissance publique neutre religieusement, n'ayant, à ce titre ni légitimité ni compétence technique pour intervenir en ce domaine. 


\section{Tentatives de structuration de l'autorité religieuse musulmane en France}

Il s'agit le plus souvent d'initiatives multiples parfois concurrentes, à l'échelle nationale comme au plan local, visant à une harmonisation progressive du champ religieux par l'institutionnalisation et le renforcement de la visibilité sociale de l'encadrement religieux musulman.

\section{Essais de cléricalisation de l'islam de France}

Ces divers processus interviennent généralement de façon concomitante aux tentatives ministérielles successives d'organisation de l'islam par le haut. Elles se sont notamment intensifiées au lendemain de l'arrivée de Charles Pasqua au ministère de l'Intérieur en 1994 et se poursuivent indirectement à propos de l'élection du Conseil français du culte musulman (CFCM). Il s'agit donc, à l'échelle nationale, d'abord de tentatives de réponses ponctuelles aux sollicitations des pouvoirs publics.

L'exemple de la tentative de mise sur pied sous la houlette du recteur de l'Institut musulman de la mosquée de Paris (IMMP), d'une véritable hiérarchie musulmane entre 1994 et 1995 en est une parfaite illustration.

Dans la Charte du culte musulman qu'il remet officiellement aux autorités, le recteur Dalil Boubakeur ne se contente pas d'user d'un vocabulaire clérical, il confectionne un organigramme précis fixant les divers niveaux de compétence et de responsabilité entre les différents cadres religieux relevant de son obédience (ethnico-politico-religieuse). C'est ainsi que sont désignés cinq Muftis régionaux représentant le recteur ayant normalement comme fonction principale de rendre des avis juridiques autorisés (fatawat) et de coordonner les actions des imams de base opérant dans une même région. Cette tentative répondait en fait à une double finalité : en premier lieu conforter aux yeux du ministre de l'Intérieur les prétentions hégémoniques de l'Institut musulman de la mosquée de Paris (IMMP) à incarner l'islam de France et à devenir l'interlocuteur officiel du ministère tout en donnant en deuxième lieu l'illusion de contrôler, par quadrillage, la majorité des mosquées de l'hexagone et d'attester d'une présence effective sur l'ensemble du territoire.

L'initiative du recteur Boubakeur rencontrera peu de succès sur le terrain. Les exemples ne manquent pas depuis le Mufti sans mosquée de Marseille, Soheib Bencheikh, qui rencontra des difficultés à se faire accepter des réseaux algériens officiels regroupés au sein de la Fédération régionale des musulmans du sud de la France, en passant par celui de Lyon Adbelhamid Chirane, dont la mosquée finira pas négocier une relative autonomie qui lui vaudra d'obtenir, après celle de Paris et le centre islamique d'Évry, l'agrément ministériel pour la désignation de sacrificateurs rituels, alors qu'auparavant elle bénéficiait de l'agrément de celle de Paris.

Abandonnant, peu ou prou, l'option des muftis, le recteur de la grande mosquée de Paris devait par la suite relancer la technique des regroupements régionaux, jadis impulsée par l'un de ses prédécesseurs, feu le recteur Abbas Bencheikh Al Hocine avec, à l'époque, le relais de l'Amicale des Algériens en France. C'est 
ainsi que le recteur de la mosquée de Paris a donné successivement sa «bénédiction » à la création, à Lyon d'un Conseil représentatif des musulmans de Rhône-Alpes (CRMRA) destiné surtout à contrecarrer l'influence locale du recteur de la grande mosquée de Lyon Kamal Kabtane et à Mulhouse, bastion de l'islam algérien en Alsace, d'un Conseil représentatif des musulmans de l'Est (CRME) face aux diverses expressions de l'islam turc et marocain.

L'initiative du recteur Boubakeur de se doter, via la Charte du culte musulman, d'un clergé docile devait en retour générer d'autres dynamiques concurrentes de la part des fédérations islamiques rivales. C'est ainsi que la Fédération nationale des musulmans de France (FNMF) liée aux intérêts marocains s'est officiellement dotée d'une Coordination nationale des imams et guides religieux (CNIGR) en 1997, alors que de son côté, l'éphémère Haut conseil des musulmans de France (HCMF) s'était doté, le 7 janvier 1996, d'un Conseil national des imams (CNI). Ce conseil s'illustrera le 7 mai 1996 par la délivrance d'une fatwa condamnant au nom de l'islam l'enlèvement des moines trappistes de Tibhéhirine (1).

L'adhésion au dit conseil était subordonnée théoriquement à certaines conditions relatives d'une part à la maîtrise d'un savoir religieux et juridique islamiques (maîtrise du Coran et de la Sunna, connaissance de l'ijma'â, des usul al fiqh, du qiyas et du 'urf...) assortie de critères de moralité publique et de critères relatifs à une bonne intégration dans la société française (maîtrise de la langue, connaissance de la société française, respect absolu de la législation nationale...) (2).

Cette énième tentative de constitution d'un corps professionnel d'imams n'avait d'autre raison d'être que de contrecarrer les ambitions hégémoniques du recteur Boubakeur et de se positionner comme une alternative crédible aux yeux du nouveau ministre Jean-Louis Debré dont le cabinet avait officieusement parrainé la création du dit Haut-Conseil devant l'incapacité chronique des responsables de la grande mosquée de Paris à élargir leur assise communautaire.

En échos, quoique intervenant plus tardivement, la Consultation lancée par Jean-Pierre Chevènement en novembre 1999 (3), poursuivie par ses successeurs, amendée par Nicolas Sarkozy, devait également se saisir de la question des imams. Fut ainsi créé, outre un groupe de travail sur les aumôniers, un autre exclusivement axé sur la question des «ministres du culte » (Groupe $n^{\circ} 3$ ). Ce groupe de travail avait pour mission de préciser quelle est la fonction de l'imam au sein des collectivités religieuses musulmanes de France, réfléchir à son statut social (notamment au regard du droit du travail et de la protection sociale) et enfin réfléchir aux diverses modalités de formation complémentaire (pour ceux déjà en activité) ou alternatives (à la formation dans les pays musulmans) pour les futurs cadres religieux.

(1) Cf. Fatwa du Conseil national des imams condamnant l'enlèvement des moines, Paris, 7 mai 1996, in Islam (Organe officiel du Haut Conseil des Musulmans de France), 1996, n 3, p 3.

(2) Cf. Déclaration du Conseil national des imams de France du 7 janvier 1996 (document dactylographié).

(3) Cf. Franck FrÉGOSI «France : le culte musulman et la République, la régulation publique de l'islam dans un cadre laïque ", in Rémy LEVEAU, Khadija MOHSEN-FINAN, Catherine WIHTOL DE WENDEN, dirs., L'Islam en France et en Allemagne. Identités et citoyennetés, Paris, Les études de La documentation française, 2001, pp 63-71 (coll. "Société »). 
Le Conseil français du culte musulman (CFCM) (4) dont $60 \%$ des membres ont été élus en avril 2003 (5) à partir des 1500 lieux de culte recensés est quasi exclusivement composé de présidents laïques d'associations et de simples fidèles ; son bureau ne comporte à l'heure actuelle aucun imam. Ce conseil a été élu au suffrage indirect (scrutin proportionnel au plus fort reste) par un collège de 4000 délégués des lieux de culte. Chaque lieu de culte retenu était pourvu d'un nombre variable de délégués au prorata de sa superficie. Des listes de candidats constituées par les principales fédérations islamiques nationales, (Grande mosquée de Paris, Union des organisations islamiques de France, Fédérations nationales des musulmans de France...) voire même des listes d'unions entre ces fédérations ou d'autres sensibilités (tabligh, islam consulaire turc, islam oppositionnel turc...) étaient proposées dans chaque région.

L'un des principaux dossiers qui lui reviendra de traiter sera paradoxalement celui de la formation des cadres religieux musulmans. Certaines des critiques formulées à son encontre, émanant de secteurs de la communauté tenus à l'écart du processus de la consultation, ciblent, d'ores et déjà, ce défaut de représentation des imams en son sein. Ce conseil appelé à représenter le culte auprès des pouvoirs publics voit donc sa légitimité contestée parce qu'il ne serait pas pourvu d'un organe religieux (6) du type conseil d'imams. Au-delà de l'aspect purement polémique, il faut reconnaître néanmoins que cette question de la formation d'imams ne devrait pas manquer d'être source de tensions supplémentaires au sein de ce conseil tant il est vrai que ses diverses composantes idéologiques ne semblent pas partager la même conception du rôle de l'imam du moins au niveau de sa formation de base, des disciplines classiques qu'il est supposé maîtriser comme de la connaissance du contexte français (7).

Parallèlement aux initiatives nationales, il faut également signaler quelques initiatives locales qui contribuent plus modestement également à la revalorisation $\mathrm{du}$ personnel religieux d'encadrement.

Il en va ainsi de la création en 1997 du Conseil des imams de Marseille et des environs (CIME) qui regroupe actuellement la majorité des imams marseillais (29), toutes mosquées, origines ethniques et sensibilités confondues (8). Ce conseil s'assigne pour objectif de mieux coordonner les actions entre mosquées à l'occasion des solennités du calendrier hégirien, l'établissement en commun des horaires des prières quotidiennes ainsi que la gestion des absences d'imams. Par le biais de ce conseil, différentes autorités religieuses musulmanes extérieures ont également été sollicitées pour émettre des avis autorisés.

(4) L'assemblée générale du CFCM comprend 200 membres (154 élus, 46 cooptés), un conseil d'administration (63 membres, 41 élus, 22 cooptés) et surtout un bureau composé de 16 membres que préside Dalil Boubakeur. En son sein la répartition des postes a été fixée en décembre 2002 lors du séminaire de Nainville-les-Roches.

(5) En fait lors de ce scrutin étaient également élus, les membres des 25 Conseils régionaux du culte musulman (CRCM) de l'hexagone qui à l'échelon local vont assurer la représentation du culte.

(6) Ces critiques émanent notamment du président du Conseil national des imams Dhaou Meskine, directeur de l'école la Réussite d'Aubervilliers.

(7) FRÉGOSI Franck, «Les filières nationales de formation des imams en France. L'institut européen des sciences humaines (Château-Chinon) et l'Institut d'études islamiques de Paris », in Franck FrÉGOSI, dir., La formation des cadres religieux musulmans en France. Approches socio-juridiques, Paris, L'Harmattan, 1998, pp 101-139 (coll. « Musulmans d'Europe »).

(8) GeISSER Vincent, "Le conseil des imams de Marseille : autonomie et pragmatisme », La Médina, avril 2001, no 7 , pp. 32-33. 
C'est principalement autour de lui que se fédèrent aujourd'hui les associations et les mosquées qui, dans le cadre du projet de construction d'une grande mosquée relancé par le maire UMP Jean-Claude Gaudin, s'opposent aux ambitions locales du "mufti" de Marseille Soheib Bencheikh, soutenu par la municipalité et les réseaux officiels algériens.

À Strasbourg, à l'initiative d'une coordination fédérant la majorité des salles de prière et mosquées de la ville, la Coordination des associations musulmanes de Strasbourg (CAMS), des réunions des différents personnels du culte (imams, aumôniers des prisons et hospitaliers) sont régulièrement organisées afin de coordonner leurs actions et de compléter leur formation.

S'agissant des populations originaires de Turquie, la situation est plus diversifiée dans la mesure où le paysage religieux reproduit les clivages politiques turcs et notamment l'opposition entre l'islam officiel de la Direction des Affaires religieuses (Ditib) (9) et l'islam oppositionnel (islamisme turc). Au sein de l'immigration turque le personnel religieux jouit d'un plus grand prestige qu'en milieu maghrébin, avec la figure centrale de l'imam-hojja (imam-enseignant,) tout à la fois responsable de la prière communautaire et enseignant de religion tant à l'échelle de l'islam officiel (Ditib-Diyanet) que des mouvements islamiques d'opposition (Millî Görüs, Suleymançi...).

On note là aussi des processus similaires de visibilisation accrue de l'encadrement religieux. À l'échelon de Strasbourg des réunions de travail ont par exemple été organisées (à l'initiative du responsable protestant des relations avec l'islam), entre des pasteurs de l'Église de la Confession d'Augsbourg d'Alsace et de Lorraine (ECAAL), luthérienne, leurs collègues de l'Église Réformée d'Alsace et de Lorraine (ERAL) et des imams de la Tendance nationale union islamique en France (TNUIF), représentation nationale de l'organisation islamiste turque Millî Görüs. Le même projet existe avec des imams maghrébins.

De son côté le Diyanet tout au long de l'année 1999-2000 a multiplié dans l'Est de la France (Vosges, Alsace...) les réunions publiques axées sur le dialogue interreligieux, en présence d'imams officiels et de représentants des autres confessions.

\section{La formation des cadres religieux à l'ordre du jour}

Cette question de la formation et de la désignation d'un personnel religieux musulman jusqu'à une date récente ne mobilisait guère les énergies en dehors de quelques cénacles d'universitaires et de chercheurs. Elle s'est néanmoins progressivement imposée comme une des interrogations majeures du devenir de l'islam en France (10) comme dans l'ensemble de l'espace européen.

(9) À l'échelle de la Turquie existe une Direction des affaires religieuses (Diyanet) rattachée directement au Premier ministère qui a en charge l'administration, la gestion et la formation des cadres religieux du culte musulman conformément aux orientations et aux principes kémalistes. Dans l'immigration, cette administration est présente sous l'appellation générique de Ditib ou Union turco-islamique d'Affaires théologiques. En France, dans le cadre de l'élection du Conseil français du culte musulman, l'islam officiel turc s'est présenté avec pour raison sociale Comité de coordination des musulmans turcs de France (CCMTF).

(10) Sorman Guy, « Des imams français », Le Figaro du 29/11/01, p 16. 
Cette évolution s'explique autant par des circonstances internes qu'internationales. L'attention particulière portée à la question du devenir du culte musulman du point de vue de ses cadres reflète, aujourd'hui, le souci des pouvoirs publics à l'échelon national - pressés de doter l'islam d'une représentation centrale du culte unifiée - d'œuvrer à l'affirmation d'un islam disposant d'un personnel d'encadrement clairement identifiable et jouissant d'une formation adaptée à ses missions et aux besoins des collectivités musulmanes de base.

Cette interrogation renvoie également à une attente réelle (bien que récente) des collectivités musulmanes elles-mêmes en matière d'imams et d'autres cadres religieux qualifiés qui soient en phase avec les milieux dans lesquels ils sont supposés intervenir notamment vis-à-vis des jeunes et par rapport à la société dans laquelle ils évoluent. Cette prise de conscience trouve dans la conjoncture internationale une légitimation supplémentaire. Il est un fait que cette question a brusquement refait surface au lendemain du 11 septembre 2001. Les diverses tentatives de reconstitution des itinéraires de certains jeunes musulmans impliqués à des degrés divers dans des réseaux actifs ou de simple soutien à des groupements islamiques radicaux ont notamment établi le rôle déterminant de certaines madrasas littéralistes au Pakistan et l'influence réelle jouée par la diffusion à grande échelle de la rhétorique wahhabite via certains prédicateurs et surtout via Internet (11).

La formation d'imams revêt donc aussi une dimension éminemment sécuritaire. Derrière l'objectif affiché de mettre sur pied une formation nationale destinée aux futurs imams, se profile l'idée de se prémunir contre toute dérive radicale en formant un personnel religieux susceptible de diffuser une vision apaisante de l'islam cadrant avec l'environnement national. Il ne faut pas perdre de vue cet aspect sécuritaire, a fortiori lorsqu'il est question de demander à l'Université de voir comment celle-ci pourrait épauler la mise en place hypothétique d'un institut islamique qui pourrait, à terme, avoir vocation à former des cadres religieux comme cela a été demandé à l'historien et arabisant Daniel Rivet chargé d'un rapport à ce sujet destiné au Ministre de l'éducation nationale.

Le ministre de l'Intérieur Nicolas Sarkozy, dans un mensuel musulman (12), a lui-même suggéré la piste de la création d'un Institut du type de l'Institut catholique recevant des subventions publiques et qui pourrait assurer une partie de la formation intellectuelle, théologique, le volet pratique, technique et pastoral devant être assuré dans le cadre d'un séminaire. Une commission interministérielle axée sur ce sujet avait été constituée. On peut par contre signaler, au sein du Conseil français du culte musulman de France, la création d'une commission sur la formation des imams.

(11) TERnissien Xavier, «L'essor des salafistes en banlieue inquiète policiers et musulmans », Le Monde du 25/01/02, p.10.

(12) Cf. La Médina, octobre 2002, no 16. 


\section{Valorisation du rôle et de la fonction de l'imam en France}

L'un des paradoxes de l'encadrement religieux musulman en France (et dans le reste de l'Europe) est le rôle prééminent qui est conféré à la charge et à la personne de l'imam, ce qui représente une situation sans précédent par rapport à celle prévalant dans le monde musulman contemporain.

\section{L’imamat en France : entre ministre du culte et médiateur socioculturel}

En effet, à la différence de ce qu'on peut observer généralement dans le monde musulman (principalement au Maghreb) où l'imam remplit plutôt un rôle secondaire, strictement limité aux aspects techniques, pratiques relatifs à l'accomplissement du culte (l'imam khatib chargé de la prédication du Vendredi, et les imams des cinq prières chargés de la direction des prières quotidiennes) dont la capacité d'autonomie, notamment en matière de prédication, est rigoureusement encadrée, contrôlée par le ministère ou la direction des Affaires religieuses, en France, on observe une tendance visant au contraire à lui conférer un rôle prééminent au sein de la collectivité religieuse musulmane.

Cette valorisation sociale de la fonction symbolique de l'imam renvoie au fait qu'en dehors du monde musulman son champ d'intervention est effectivement plus large que la sphère stricte du culte quotidien et hebdomadaire. Il tend en fait à cumuler plusieurs fonctions au plan religieux, social et civil pour ne pas dire civique. C'est ainsi qu'il prend souvent en charge l'éducation des plus jeunes, s'occupe de l'accompagnement spirituel des fidèles hors des lieux de cultes et se transforme, à l'occasion, en médiateur interculturel et social dans certains quartiers. On a ainsi pu observer à l'occasion de certaines tensions dans des banlieues survenues à la suite de bavures policières que l'imam se faisait alternativement le relais de la colère des familles dont l'un des enfants venait d'être abattu au terme d'une échauffourée avec les forces de l'ordre tout en s'imposant comme un modérateur social lançant des appels au calme.

Autre exemple, lors des obsèques du jeune Khaled Kelkal, recherché pour avoir participé à des attentats et abattu par les forces de l'ordre le 29 septembre 1995, la cérémonie religieuse fut officiellement présidée par le Mufti de Lyon en personne Abdelhamid Chirane accompagné de son homologue de Vénissieux Kamal Mansour. Leur présence visait autant à apporter un surcroît de réconfort aux proches du disparu, que rappeler à ces jeunes que des imams actifs sur le terrain (notamment Kamal Mansour aumônier des prisons) étaient à leur disposition comme recours afin d'éviter toute dérive du type de celle dans laquelle avait pu sombrer Khaled Kelkal.

Nombre d'interrogations demeurent cependant quant aux motivations réelles de cette valorisation de la personne de l'imam qui peut parfois sembler très artificielle lorsqu'on prend en compte le fait que la plupart des mosquées et des grandes fédérations musulmanes de France ne sont pas présidées par des responsables en charge directe du cultuel, mais bien par des "laïcs », présidents d'associations qui n'ont pas suivi une formation classique spécialisée en sciences islamiques. Il convient de relever, à titre d'illustration, que le groupe de travail qui avait été constitué dans le 
cadre de la Consultation sur l'islam (l'Istischara) sous le vocable « Ministres du culte » ne comprenait tout au plus qu'un membre permanent qui assurait de façon régulière la fonction d'imam à Mantes-la-Jolie, les autres membres étaient soit des membres de professions libérales (avocat, médecin), des responsables associatifs, ou des universitaires.

Sans doute faut-il voir dans cette valorisation de la figure de l'imam l'expression d'une adaptation progressive à un environnement culturel marqué par le christianisme dont la figure religieuse historiquement dominante fut longtemps celle du clerc, du prêtre. Cette évolution exprime l'une des principales mutations récentes du leadership religieux musulman en France.

\section{Les nouveaux clercs séculiers de l'islam}

La personnalité de l'universitaire suisse d'origine égyptienne Tariq Ramadan, par son aura auprès du public jeune musulman et par son discours illustre dans l'espace européen francophone l'émergence d'un nouveau type de leadership et la déclinaison de l'autorité religieuse musulmane sur un mode séculier qui se distingue très nettement des formes plus conventionnelles. En effet, là où les clercs traditionnels musulmans ('alim, mujtahid) avaient tendance à asseoir leur autorité religieuse en mettant en avant une habilitation religieuse conférée par leurs pairs (al 'ijaza), une connaissance transversale à la fois des textes fondamentaux (Coran, Sunna) et de la jurisprudence islamique (de sa méthodologie comme de sa théologie) les nouveaux clercs du type de Tariq Ramadan mettent plutôt en avant un vécu quotidien, une expérience personnelle (celle d'un musulman européen), et indirectement dans son cas, une lignée familiale (figure de son grand-père Hassan Al Banna, fondateur de l'association égyptienne des Frères Musulmans en 1929 et celle de son père Saïd Ramadan, co-fondateur de la Ligue Islamique Mondiale (LIM). Sans oublier cet autre ressort ô combien déterminant en ce qui le concerne : une remarquable rhétorique, servie par un style pédagogique et un indéniable charisme.

Ce que nous relevons à propos du cas Tariq Ramadan se retrouve peu ou prou avec d'autres figures musulmanes d'Europe comme des pays islamiques. Olivier Roy fait en effet la même constatation à propos de quelques figures emblématiques de l'islamisme radical comme Oussama ben Laden mais aussi Abu Hamza al Misri, de la mosquée de Finsbury Park à Londres qui n'ont jamais suivi les cursus classiques en formation islamique, et qui fustigent les clercs traditionnels jugés trop timorés (13). Il convient d'observer que dans les premiers écrits de Tariq Ramadan l'argumentaire strictement de nature théologique n'était pas prédominant, cela s'explique en partie par l'auditoire, bien qu'éduqué dans sa grande majorité, le public visé semble ne pas avoir une connaissance approfondie du patrimoine religieux arabo-musulman, ni maîtriser complètement les catégories de la pensée théologique musulmane. De la même manière, son auditoire ne fait pas preuve d'une connaissance poussée de l'histoire des idées dans le monde arabo-musulman,

(13) Roy Olivier, L'islam mondialisé, Paris, Seuil, 2002, pp 87-99 (coll. « La couleur des idées »). 
ce qui laisse le champ libre aux nouveaux clercs pour présenter leur vision de l'histoire de tel ou tel courant de pensée (celui du réformisme et celui des Frères Musulmans) au prix de quelques raccourcis et moyennant quelques réécritures destinées à des fins hagiographiques (14) que les spécialistes se doivent de relever sans pour autant instruire à charge le procès d'un acteur central de l'islam européen (15).

\section{Structure formelle de ce leadership}

Le discours de ce type de leader d'opinion présente quant à son aspect formel les trois caractéristiques suivantes : il emprunte au registre du témoignage (à la fois celui d'un musulman européen parmi d'autres, et celui de l'héritier d'une lignée familiale militante au nom de l'islam), il se présente comme un discours résolument pédagogique à destination principalement des jeunes musulmans, il entend proposer une articulation alternative entre foi et action sociale.

Le discours de Tariq Ramadan ne se décline pas sur le registre du discours savant, de l'expert, mais plutôt sur celui de l'expérience individuelle, du vécu, du témoignage. «Ce n'est ni une référence morale, intellectuelle, sociale (...), je vous parle en tant que musulman vivant en Suisse, je suis d'ici » a-t-il souvent l'habitude de dire en introduction à ses conférences. La mise en avant de sa qualité de musulman européen lui permet ce faisant de se démarquer aussi bien des clercs musulmans étrangers à la société européenne, française, que des universitaires d'origine musulmane, bref de toute élite constituée, trop décalée par rapport à la masse des fidèles et à leurs attentes. Le témoignage de l'expérience personnelle se complète toujours chez lui logiquement d'une référence appuyée à la lignée familiale, à l'engagement militant au nom de la da'wa islamique tout d'abord de son grand-père Hassan Al Banna (1906-1949) via l'association politico-religieuse des Frères Musulmans dont il fut le fondateur et qui, peu ou prou, a constitué l'une des principales matrices des grands mouvements islamistes contemporains (16). Référence également à son père, Saïd Ramadan qui, durant les années cinquante du sein de la mouvance des Frères Musulmans, mena une lutte active contre la gauche nationaliste arabe via la Ligue Islamique Mondiale, dont il fut l'un des acteurs majeurs. Replié dans son exil genevois, après sa rupture avec les Saoudiens, Saïd Ramadan se fit l'avocat zélé de la cause de l'islam en Occident via sa revue $\mathrm{Al}$ Muslimoun (Les Musulmans).

Le discours de Tariq Ramadan se décline aussi sur un mode pédagogique, qu'il s'agisse d'inviter les jeunes musulmans à mieux connaître l'histoire des sociétés occidentales et de la laïcité en particulier, ou qu'il faille justifier d'un point de vue islamique la licéité de la vie des musulmans en Europe. Le discours se veut posé, méthodique même si parfois il procède à une lecture sélective de l'histoire. Il s'agit d'édifier, d'amener son auditoire à la fois à prendre conscience de son «mal-être »,

(14) En ce qui concerne Ramadan, voir Franck FRÉGOSI, « Tariq Ramadan ou les habits neufs d'une vieille rhétorique », Chemins de Dialogue, 1999, no ${ }^{\circ}$, pp.125-151.

(15) Les prétoires et certaines pseudo expertises post 11 septembre 2001, tout comme les discours de louange déplacés, cadrent assez mal avec le souci de l'analyse et de la rigueur scientifique.

(16) Cf. Roy Olivier, Généalogie de l’islamisme, Paris, Hachette, 1995, p. 35 (coll ; «Questions de société »)). 
de son oubli d'identité qui renvoie d'une part à l'oubli de Dieu et d'autre part à l'attrait du modèle occidental. Ramadan en même temps invite les musulmans à ne pas se complaire dans cet état, il les incite avec des accents réformistes à se ressaisir en redécouvrant la fierté d'être musulman, ce qui implique à la fois une fidélité à une dimension spirituelle (« lien à la transcendance ») et un engagement social fort («l'islam vecteur de justice sociale»). Ceci l'amène à définir les contours «d'une religiosité citoyenne » (17). Ce mode spécifique de présence dans la cité et d'action sociale repose sur une articulation entre une appartenance forte à l'islam et un engagement citoyen dans la sphère publique. « Il n'y a pas de conscience islamique sans conscience sociale (...), écrit-il, pas de conscience sociale sans conscience politique »(18). Pour lui l'identité islamique quel que soit le contexte se déploie autour de quatre axes qui sont la foi (conviction, pratique, spiritualité), l'intelligence des textes et du contexte (compréhension des sources et du cadre environnant), la transmission (éducation), l'action et la participation (être social) (19). Cette dernière dimension constitue la plénitude de l'être musulman, son accomplissement. La pleine intégration dans la société pour les musulmans passe notamment selon Tariq Ramadan par «l'actualisation d'une stratégie d'intervention sociale » spécifiquement islamique ce qui pour lui revient à reconsidérer le sens et la place de la sharî'a dans le quotidien des musulmans en Europe.

Sur ce dossier sensible de la législation islamique, loin des prises de positions radicales de son frère Hani (20), Tariq Ramadan se montre à la fois prudent et pragmatique; prudence sans doute dictée par le caractère délicat d'une référence à la sharî' $a$ hors du monde musulman et dans le contexte international tendu de l'après 11 septembre, et pragmatisme dans sa manière d'assigner comme finalité sociale à la législation islamique l'établissement de la justice sociale, là où d'aucuns auraient plutôt attendu une définition essentialiste. On perçoit dans ce discours la difficulté constante chez cet auteur à devoir concilier une présentation de l'islam comme spiritualité sans pour autant se défaire d'une tendance à ériger l'islam en une identification globale et universaliste qui dépasse le simple cadre confessionnel, « l'islam est plus qu'une religion » selon lui !

\section{Faisceaux de légitimités des nouveaux clercs}

Ce type de leader semble porteur de plusieurs faisceaux de légitimités.

Il y aurait lieu d'en distinguer au moins quatre :

- une légitimité de type charismatique (la plus évidente) qui renvoie aux qualités « exceptionnelles » de la personne, à son talent oratoire, à sa rhétorique claire, son style pédagogique ;

(17) FRÉGOSI Franck, « Les contours discursifs d'une religiosité citoyenne : laïcité et identité islamique chez Tariq Ramadan », in Felice Dassetto, dir., Paroles d'islam. Individus, sociétés et discours dans l'islam européen contemporain/Islamic Words. Individuals, Societies and Discourse in Contemporary European Islam, Paris, Maisonneuve \& Larose/European Science Foudation, 2000, p. 219.

(18) Ramadan Tariq., Les musulmans dans la lä̈cité. Responsabilités et droits des musulmans dans les sociétés occidentales, Lyon, Tawhid, 1994, p. 46.

(19) Ramadan Tariq, Les musulmans d'Occident et l'avenir de l'islam, Paris, Sindbad, Actes Sud, 2003, pp. 140-150 (coll. «L'actuel»).

(20) Ramadan Hani, «La charia incomprise », Le Monde du 9/09/02. 
- une légitimité héritée de type historique qui nous plonge dans la lignée familiale de Hassan Al Banna, lignée à la fois croyante et militante. Par ce biais, le discours de Tariq Ramadan s'intègre dans une continuité historique et idéologique illustre ;

- une légitimité expérientielle de terrain au sens où son autorité ne repose pas prioritairement sur sa position statutaire dans le champ de production du savoir islamique, mais s'enracine précisément dans une expérience personnelle, un engagement et un vécu (21);

- une légitimité médiatique enfin : l'audience actuelle de Tariq Ramadan semble en effet largement dépasser le strict cadre des milieux jeunes musulmans pour inclure certains milieux intellectuels (anciens de l'extrême-gauche) et journalistiques (Le Monde Diplomatique, Le Monde des Débats...), universitaires, religieux catholiques notamment (compagnons de route) et le monde laïque militant (Ligue Française de l'Enseignement...) et associatif (Ligue de Défense des Droits de l'Homme). Ce qui me conduit à dire, pour reprendre des typologies jadis utilisées à propos du leadership immigré (22), qu'à sa manière Tariq Ramadan combine un double leadership: des faisceaux de légitimités internes et de légitimités externes. Il représente selon l'expression empruntée à John Higham (23) un « internal leader» dans le sens où il s'agit bien d'un leader issu de la collectivité musulmane européenne (même s'il bénéficie de la légitimité reçue de son père et de son grand-père qui, elle, est externe à ce groupe) et en même temps c'est un leader projectif dont l'audience s'étend au-delà de son groupe d'identification originel, confessionnel.

\section{Le jurisconsulte et la sharî'a de minorité}

Notre analyse du champ de l'autorité islamique en France ne serait pas complète si elle ne prenait pas en compte une troisième figure émergente, celle du jurisconsulte.

Comme pour l'imam, il s'agit d'une figure classique du champ religieux islamique. À l'exception des prédicateurs de l'islam politique, l'imam en contexte musulman est généralement cantonné à l'activité liturgique, à l'encadrement technique de la dévotion quotidienne; il est stricto sensu un ministre du culte. Le

(21) Habituellement selon le cadre islamique classique, la source d'imitation par excellence pour tout fidèle musulman doit être la figure du Prophète; dans le cas de la figure charismatique de Tariq Ramadan il convient de soulever, que l'on se trouve parfois confronté chez ses auditeurs à un glissement de référent, de l'archétype de la figure du Prophète à celle du musulman Tariq Ramadan érigé en modèle pour les musulmans vivant en Europe. Nous avons pu observer ce phénomène à partir de témoignages de jeunes musulmans turcs faisant état parmi les auditeurs d'une survalorisation de la personne du conférencier Tariq Ramadan au travers d'une formule du type: «Nous croyons nous à l'islam de Tariq Ramadan. »

(22) Conseil européen des fatwas et de la recherche, Recueil de fatwas. Avis juridiques concernant les musulmans d'Europe, série no ${ }^{0}$, Lyon, 2002, pp. 54-55.

(23) Voir Marco MARTiniello, Leadership et pouvoir dans les communautés d'origine immigrée, Paris, CIEMI-L'Harmattan, 1992, p. 54 (préface d'Albert Martens) (coll. " Migrations et changements »). 
jurisconsulte, incarne lui, davantage la figure du clerc, du docteur de la loi ('alim), celui dont la compétence professionnelle lui permet d'interpréter les textes (mujtahid) et de rendre des avis juridiques autorisés (fatwa).

En Europe, en l'absence d'institutions islamiques susceptibles de réguler le champ religieux islamique, il va de soi que la figure du jurisconsulte reste pour l'heure encore largement une figure d'importation à laquelle les musulmans peuvent avoir accès via Internet (24) ou plus ponctuellement lors du séjour et de la visite de certains ulémas de renom dans les communautés immigrées à l'occasion de congrès islamiques du type de celui organisé annuellement par l'UOIF ou de colloques organisés par des centres islamiques comme celui de la mosquée $\mathrm{Ad}$ dawa du XIX ${ }^{\mathrm{e}}$ arrondissement de Paris.

Pour autant il convient de noter que l'enracinement progressif de l'islam dans l'espace européen dans un environnement non musulman et sécularisé ne va pas sans susciter des interrogations parmi les musulmans européens les plus observants. Cela a conduit certaines organisations islamiques comme l'Union des organisations islamiques en Europe à donner naissance en 1997 à un Conseil européen des fatwa et de la recherche. Ce conseil, dont le siège est en Grande-Bretagne, afin de répondre aux multiples demandes depuis 1998, s'est doté de deux commissions régionales : l'une en France et l'autre en Grande-Bretagne. Composé d'une trentaine de membres et présidé par le cheikh qatari d'origine égyptienne Yussef $\mathrm{Al}$ Qaradawi, ce conseil se fixe, en interne, pour objectif d'unifier les avis juridiques destinés aux communautés musulmanes d'Europe. Il se spécialise donc dans la jurisprudence des minorités (fiqh al-aqalliyyât) musulmanes vivant en dehors du monde musulman.

Par rapport aux gouvernements européens il entend être reconnu comme l'instance officielle en matière de production et de diffusion du savoir sur le droit musulman et recommande aux musulmans résidant en Europe d'œuvrer afin que l'islam soit une religion reconnue et que soit également reconnus «à l'instar des autres minorités religieuses, tous leurs droits relatifs à l'organisation de leur statut personnel en matière de mariage, de divorce et d'héritage » (25).

La France occupe en ce domaine de l'adaptation de la législation islamique au contexte national une position particulière en raison d'une part de son régime de laïcité, d'autre part de l'importance numérique de la communauté musulmane qui y réside. Aussi n'y a t-il rien d'étonnant à ce que l'un des principaux canonistes musulmans qui depuis l'Europe aborde cette question dans le détail y exerce son «ministère ». Il s'agit de Tareq Oubrou imam et recteur d'une mosquée de Bordeaux. Cet imam d'origine marocaine, qui préside l'association des imams de France (26), s'est notamment illustré en élaborant le concept et la théorie de "sharî̀'a de minorité".

(24) Voir notamment le site http: //www.islamophile.org qui comprend une banque de fatwa consultable en langue française par un index thématique ou un index par mufti, ou bien via le site officiel de l'Union des organisations islamiques de France (UOIF), http : //www.uoif-online.com, l'internaute a la possibilité de soumettre lui-même une question au Conseil européen de la fatwa.

On peut également se référer aux divers sites salafistes suivants qui diffusent les fatwas émises par les cheikhs saoudiens les plus rigoristes : http : //www.fatwas.online.fr, http : //www.soubhannallah.com, http : //www.sounnah.free.fr, http : //www.al.baida.online.fr.

(25) Conseil européen des fatwas et de la recherche, Recueil de fatwas. Avis juridiques concernant les musulmans d'Europe, série no 1, Lyon, 2002, p 39.

(26) Association rattachée à l'Union des organisations islamiques de France (UOIF). 


\section{Orthodoxie et orthopraxie minimales pour islam minoritaire}

À la différence des groupes marginaux (Al Muhajirun en Grande-Bretagne et des salafistes) un certain nombre de décisionnaires orthodoxes (par réalisme mais aussi par conviction intime !) ont élaboré dans le contexte européen une "théorie restreinte de la sharî‘a » (27), qui s'efforce de prendre acte de l'impact de la sécularisation sur la façon dont les musulmans peuvent vivre l'islam en situation minoritaire sur un mode à la fois cultuel ('ibâdât) et éthique (akhlâq).

Sans nullement remettre en cause le caractère universaliste contenu dans le message islamique, Tareq Oubrou entend ainsi défendre l'idée selon laquelle si les musulmans vivant en France peuvent parfaitement pratiquer en toute quiétude l'intégralité des préceptes de la religion musulmane (al 'ibâdât), s'agissant des règles et des dispositions en matière de relations sociales (al mu'âmalat), ces dernières doivent nécessairement faire l'objet d'une adaptation au contexte culturel et juridique français. Il en vient ainsi à prôner en matière dogmatique et cultuelle une " orthodoxie minimaliste " qu'il entend conjuguer avec une " éthicisation de la sharî'a ». Il ne s'agit pas pour lui de renoncer à toute référence globale à la sharî'a mais simplement de constater et de formaliser le fait qu'en Europe, celle-ci se restreint aux questions de pratiques cultuelles, de principes moraux. Les autres domaines doivent faire l'objet soit d'adaptations circonstancielles par le procédé de la fatwa ou d'une interprétation sur un mode éthique via le recours aux fictions canoniques (fiqh al-hiyyal). "L'éthicisation de la sharî'a vise en fait à conférer la légalité morale islamique à certains comportements du musulman en intégrant le droit français dans le métabolisme de la sharî'a. Il exclut le droit de la sharî’a, en le réduisant à la seule dimension morale » (28).

La fatwa permet quant à elle « de contextualiser la sharî‘a et par conséquent de proposer différentes expressions de la visibilité de l'islam. » (29) Ceci revient en fait à définir les contours théoriques d'une législation islamique de portée nationale à partir de laquelle est possible l'observance d'une orthopraxie moyenne adaptée au contexte sécularisé et laïque français. « À chaque époque écrit Tareq Oubrou, à chaque contexte le canoniste se représente a priori une orthodoxie moyenne autour de laquelle il construit sa lecture de la sharî'a (...) il y a un seuil ou un niveau moyen de pratique religieuse pour chaque époque (...) il existe une forme de religiosité minimum nécessaire pour une communauté musulmane donnée dans des circonstances données. La fatwa commune devra à ce titre énoncer un ensemble de devoirs islamiques qui constituent ce quantum minimum au-dessous duquel nous ne pouvons descendre sans manquer à notre devoir religieux » (30). Ce travail de relativisation de la sharî'a s'accompagne logiquement d'une valorisation de la fonction et du statut de faqih (jurisconsulte) et de mufti à qui revient notamment selon Oubrou la tâche de formuler les fatwas susceptibles de favoriser le maintien de cette religiosité minimum.

(27) Oubrou Tareq, « La sharî‘a de minorité : réflexions pour une intégration légale de l'islam », in Franck FrÉGOSI, dir., Lectures contemporaines du droit islamique. Europe-Monde arabe, Presses Universitaires de Strasbourg, 2004 (à paraître).

(28) Voir Ramadan Hani, op. cit.

(29) Ramadan Hani, op-cit.

(30) Ramadan Hani, op-cit. 
Là où l'imam doit exhorter par la prédication, en toute circonstance, et en tous lieux, les fidèles à respecter par exemple l'observance des cinq prières quotidiennes, le mufti doit lui, à partir de sa double maîtrise de l'outil canonique islamique et de sa connaissance du droit français (et la prise en compte d'un droit européen en construction !) parvenir à élaborer une théorie adaptative du canon islamique spécifique au contexte sécularisé prévalant en France. Là où le clerc séculier, qui lui aussi insiste sur l'intelligence du contexte mais met davantage l'accent sur l'engagement social et citoyen du musulman, qui est constitutif de l'être musulman en général, le jurisconsulte, reste davantage préoccupé par la dimension normative de l'être islamique et son adaptation au contexte environnant et à la situation minoritaire.

En dépit de cette divergence de vue ces deux types d'attitudes ont une finalité commune qui est de définir un "seuil d'islamité minimale », sociale ou juridique, en dessous duquel le musulman ne peut descendre au risque de produire un « islam au rabais » ou de se dissoudre totalement et, partant, de cesser d'être musulman. «L'enjeu, soyons clairs, consiste, comme l'écrit Tariq Ramadan, à se donner les moyens de rester musulmans tout en vivant dans de nouveaux environnements, fondés sur des rapports au religieux, à la pratique et à la morale, que les musulmans ne connaissaient pas dans leur pays d'origine. » (31)

\section{En guise de conclusion}

Le paysage religieux musulman en France, du point de vue de son encadrement et de son leadership, se caractérise par la très grande diversité des types et des sources d'autorités religieuses qui s'expriment de façon plus ou moins concurrentielle entre elles, ainsi que par l'étendue de leurs sphères d'intervention respectives. Celles-ci se déclinent du culte quotidien, à la socialisation religieuse, à la médiation culturelle en passant par l'action sociale voire la médiation politique avec les autorités locales.

$\mathrm{Au}$ sein de ce paysage pluraliste, nous assistons cependant à sa cristallisation autour de trois figures principales : celle de l'imam dont la sphère d'action dépasse le simple domaine cultuel pour inclure celui du socioculturel, la figure encore hégémonique du leader charismatique jeune musulman, de l'intellectuel militant et progressivement celle du jurisconsulte, du canoniste qui s'efforce d'accompagner l'intégration progressive des musulmans dans l'espace européen par un travail de relativisation de la législation islamique en vue de son adaptation à la vie en Europe.

Ces diverses expressions du leadership islamique apparaissent à la fois comme concurrentes dans leur commune propension à produire du sens pour le devenir des musulmans vivant en France et étroitement complémentaires dans leurs modes spécifiques d'énonciation.

(31) Ramadan Tariq (Préface) Conseil européen des fatwas et de la recherche, op.cit., pp 12-13. 
À l'exemple de ce que l'on observe déjà dans l'ensemble du monde musulman, les femmes, hors des sociétés musulmanes semblent, pour l'heure, n'occuper qu'une position marginale dans le registre de l'autorité religieuse. Leur action se limite le plus souvent à la constitution d'un tissu associatif féminin évoluant plus ou moins en marge des grandes fédérations islamiques. En son sein, elles s'attachent à aborder les problèmes spécifiques liées à l'identité féminine islamique en dispensant notamment un savoir islamique de base articulé autour de la bipolarité structurante du hallal et du haram, du licite et de l'illicite dans l'islam destiné à leur fournir des repères afin d'être de «bonnes musulmanes » pratiquantes (32).

Il est encore trop tôt pour considérer cette répartition des rôles comme définitive, a fortiori au sein d'un paysage islamique en mutation, confronté à une pluralité d'acteurs qui au gré des circonstances de leur positionnement social et de leur genre font un usage social différent du référent islamique. Celui-ci peut aussi bien être mobilisé alternativement dans une perspective résolument conservatrice (intellectuelle, religieuse, politique...), réformatrice (néo-réformisme, islam libéral) voire comme vecteur d'une logique de contestation par exemple d'un certain ordre mondial (islam alter mondialiste) ou d'une lecture exclusivement masculine de l'islam (33).

\author{
Franck FRÉGOSI \\ Société, droit et religion en Europe \\ Université Robert Schuman-CNRS - Strasbourg
}

(32) «Louisa Latrache, femme musulmane, militante », Interview par Abdallah Sindonino, in Islam de France, 1999, nº 5, pp 125-133.

(33) Voir Weibel Nadine B., Par-delà le voile. Femmes d'islam en Europe, Bruxelles, Éditions Complexe, 2000 (coll. «Les dieux dans la cité »). 
Résumé

Cet article est axé sur la question des contours pluriels de l'autorité religieuse dans le paysage islamique français. Cette question revêt une importance sociale déterminante de nos jours à l'heure où les pouvoirs publics se sont engagés dans une politique volontariste de régulation du fait islamique dont l'élection en avril $2003 d u$ Conseil français du culte musulman a été l'une des manifestations majeures.

Cet article présente un aperçu global des diverses modalités de déclinaison de l'autorité religieuse islamique au travers de l'étude des trois principales figures islamiques contemporaines de l'imam, du conférencier et du jurisconsulte.

\section{Abstract}

This paper deals with the question of religious authority in the context of muslim community in France, in a non muslim and a deeply secularized society. This question is today debated and linked with the new institutionnal context of a strongly voluntarist public regulation of islam but also with the question of the public influence among young muslims of a new type of charismatic muslim leader like Tariq Ramadan.

The main topic of this paper is to give a general presentation of the situation of the religious islamic field in France: initiatives towards the clericalisation of imams, the different modes of variation of religious authority and of islamic leadership. This study will focus on three central figures of the imam, the muslim youth leader and the muslim jurisconsult.

Resumen

Este artículo se centra en la cuestión de los contornos plurales de la autoridad religiosa en el paisaje islámico francés. Esta cuestión reviste una gran importancia en nuestros días, en el momento en que los poderes públicos han emprendido una política voluntarista de regulación del hecho islámico, de la cual la elección, en abril de 2003, del Consejo francés de culto musulmán ha sido una de las manifestaciones mayores.

Este artículo presenta una perspectiva global de las diferentes modalidades de declinacióde la autoridad religiosa a través del estudio de tres figuras islámicas contemporáneas : la del Imam, la del conferencista y la del jurisconsult. 